Journal of Social Sciences 8 (3): 372-380, 2012

ISSN 1549-3652

(C) 2012 Science Publications

\title{
Finding What You Expect to See: Theoretical Modeling in Psycholinguistics
}

\author{
Kathleen Ahrens \\ Language Centre, Hong Kong Baptist University, \\ 224 Waterloo Road, Kowloon Tong, Kowloon, Hong Kong
}

\begin{abstract}
Problem statement: Theories of language processing rely upon experimental evidence to support or reject their hypotheses. Yet it is often the case that conflicting theories flourish alongside each other for decades, with voluminous experimental evidence to support their respective hypotheses. Approach: In this study, I suggest it is imperative for researchers to move beyond their own experiments and results to embrace different methodologies. With respect to language processing in particular, it is reasonable to suggest that the brain needs to perform a variety of tasks under a variety of conditions (or contexts). Results: Looked at in this way, the varying results do not suggest that one or the other theory is right and the other wrong. Conclusion: Instead, it suggests that conflicting theories are supported by a plethora of data precisely because different contexts require the brain to process language in different ways.
\end{abstract}

Key words: Language processing, lexical ambiguity resolution, conceptual mapping model, on-line sentence processing, methodology

\section{INTRODUCTION}

Language researchers often group themselves into different camps, just like hikers from different countries at a campground in the Appalachian Mountains, who pitch their tents in a particular area, venturing outside their camp to use the communal facilities or to buy necessities. When they venture out, they encounter other hikers. They are polite; they may chat, sit down and have a meal. They may even sing a few songs together. But at the end of the day, they return to their own camp and the next day they pack up and they follow their own group on the trail that that group has chosen to hike. They may meet up with a group one or two more times as they hike the trail. They may not. At the end of their hiking journey, they pack up and go back to their respective countries.

This is analogous to language researchers who meet each other at conferences and workshops. They may listen to each other's papers; they may discuss each other's findings; they may even agree to disagree. At the end of the conference, however, they go back to their own labs. Of course, at some larger conferences, even this degree of interaction may not happen. Each group may listen only to papers that are relevant to themselves. Oftentimes, it is not premeditated disregard for other's work; it is instead due to time constraints: when there is so much to listen to and so little time, naturally one will listen to talks one deems most relevant.
In this study, I will look at two long-term issues in the psycholinguistic literature. The first issue involves the modularity-interaction debate with regard to lexical ambiguity resolution. In this case, it will be shown that experiments in which the participant is expected to problem-solve return a result that supports an interaction hypothesis, while those experiments with similar types of stimuli in which the participant is asked to complete a task tangential to the actual sentence being read return a result that supports a modularity hypothesis. Thus, tasks that ask for problem-solving in return find for interactivity, whereas tasks that ask the participants to perform two tasks automatically find for modularity.

The second issue examines two models of metaphor processing: the Attributive Categorization model (AC model) and the Conceptual Mapping model (CM model). The AC model (McGlone, 1996; Glucksberg et al., 1997; Keysar et al., 2000; Jones and Estes, 2005) argues that conceptual metaphors are not processed on-line in real time but are instead understood later in the comprehension process, while the CM model (Nakayama, 2002; Gong and Ahrens, 2007) argues that conceptual metaphors are, in fact, interpreted on-line. In this case, it will be shown that when participants are expected to read sentences one at a time in a paragraph, the results show that no activation of a conceptual metaphor occurs, which supports the attributive categorization hypothesis; 
however, when the same sentences are read one at time within one paragraph, activation of a following conceptual metaphor occurs, supporting the conceptual mapping model. Thus, the paragraph form allows for a build-up of the conceptual mapping, while reading sentences one at a time does not.

What these two sets of comparisons demonstrate is how the methodology (i.e., the context of the experiment) and the stimuli chosen constrain the potential results. In this way, researchers are able to find what they expect to see. I suggest that it is imperative for researchers to move beyond their own experiments and results to embrace different methodologies and different stimuli, thus providing different contexts in which to test their hypotheses and to expand the data set that they can explain with their models.

Lexical ambiguity resolution: Lexical ambiguity resolution (Altarriba and Gianico, 2003 for a review) has been an area of interest for researchers for the past thirty years, in part due to the implications the results have four models of cognition and in part due to the apparent simplicity of experiments that can be run to test the various hypotheses related to the various cognitive models. In what follows, I will first discuss types of ambiguity and then present the major models. Next, I will point out that the apparent simplicity of the experiments in fact revolves around several taken for granted aspects that all, implicitly or explicitly, have to do with context: modality, task, visual probe position and timing.

Types of ambiguity: Words often have multiple senses. These senses may vary within or across a lexical category. For example, 'rose' varies between the nominal sense of a flower and the past tense of the verb 'rise' (example 1a and 1b):

(1a): The rose she handed to him pricked his thumb when he took it from her.

(1b): The sun rose over the mountaintop, sending bright rays into the eyes of the waiting onlookers.

In example (2), the ambiguity occurs within the same lexical category, as 'glasses' can refer to either eyeglasses or drinking glasses.

(2) Please hand me the glasses.

What is interesting in both cases is that it is rare for one sense to be confused with the other. This may be because syntactic information helps select the appropriate meaning, or because collocation patterns prefer one reading over another, as in the case of (2) versus (3), where (3) is more likely to refer to eyeglasses and (2) is more likely to refer to drinking glasses.

(3) Please hand me my glasses.

Thus, contextual clues within the sentence itself direct the interpretation to the one that allows for the most likely interpretation of the utterance. Of course, puns are the exception to this rule, as the point of a pun is to sustain ambiguity for a humorous effect, as in (4).

(4) After the ship crashed, the captain headed straight for the port.

In (4), it is ambiguous as to whether the captain is reaching for the liquor bottle or is steering the ship toward the harbor. Note that sustained ambiguity for the purpose of humor is different from vagueness, as in the case of (5), where what 'glasses' is referring to is unspecified in this sentence.

(5) The glasses fell on the floor.

Research in examining how lexical ambiguity is resolved in on-going sentence processing usually focuses on the type of ambiguity found in the example (4), what Ahrens et al. (1998) call 'active complexity (Research that looks at what Ahrens et al. (1998) refer to as latent complexity, as in example (5), is usually examined with the aid of lexical decision tasks run independent of on-going sentence processing (for example, see Lin and Ahrens, 2010)'.

Cognitive models: Hypotheses concerning how cognitive processing proceeds can be categorized broadly into two main types. In the first type, the processing system involves a set of processing modules that are functionally autonomous. This is called the modularity hypothesis (Fodor, 1983). Under the assumptions inherent in this model, two of which are automatic and autonomous, a module computes the same output to a particular input, irrespective of the context surrounding the input. In the second type, processing modules can interact and share information across levels (Marslen-Wilson and Tyler, 1980; McClelland and Rumelhart, 1981; McClelland, 1987). One advantage to this system is that feedback permits higher-level constraints to guide lower-level processing.

These theories of cognitive processing have been examined at the level of language processing by testing whether sentence-level contextual information can influence the postulated lower-level of lexical access. A context-independent model predicts that preceding sentential context will not influence lexical access. Two accounts arise as a result of this prediction: the first, known as the multiple-access account, is that all meanings of a word are accessed irrespective of the preceding context (Onifer and Swinney, 1981; 


\section{J. Social Sci., 8 (3): 372-380, 2012}

Swinney, 1979); and the second is that the most frequent meaning is accessed irrespective of context (ordered-access model). If that meaning does not fit the context, then the next most frequent meaning is accessed until a meaning is found that fits in with the context (Hogaboam and Perfetti, 1975) (The issue of which meaning is more likely to be available given a particular physical environment, regardless of the 'overall frequency,' has not yet been discussed. For example, the meaning of 'mouse' as a peripheral device may be secondary to its meaning as a mammal, but participants sitting in front of a computer in an experimental setting may be more primed for the secondary meaning).

The context-dependent, or direct access, model predicts that sentential context will provide enough information that only the contextually appropriate meaning will be accessed (Martin et al., 1999; Vu et al., 1998). This preceding context information will be enough for the processor to identify the correct meaning, even if the sense is of very low frequency.

In recent years, a third model has been proposed that suggests that both contextual and lexical frequency factors influence lexical access, such that context facilitates the activation of a contextually appropriate meaning but does not inhibit high frequency meanings. This predicts that when the context is biased toward the more frequent meaning, only that meaning will be accessed. However, when the context is biased toward a less frequent meaning, both the more frequent meaning and the context appropriate (but less frequent) meaning will be accessed (Duffy et al., 1988 [Reordered Model]; Giora, 1997; 1999; 2003; Peleg et al., 2001; Noveck and Sperber, 2006 [Graded Salience Hypothesis]).

All three models receive support in the literature at the expense of alternate models. Thus, the question arises: How can a model be right and wrong at the same time? The answer, I will suggest, has to do with subtle variations in the context of the entire experiment, including the modality of presentation, the type of task that is required of the participants, the position of the visual probe and the presentation length of the probe.

Modality: Lexical ambiguity resolution experiments usually involve a complete visual presentation, or an audio presentation of a sentence complemented by a visual presentation of a target word. A purely visual presentation is used in reading tasks, such as reaction time experiments, event-related potential experiments and eye-tracking experiments. In visual presentations, the words may appear one at a time in the center of the screen for a designated period of time, or they may appear at the left of the screen and then move across to the right to mimic reading. The words may remain on the screen or disappear after a certain amount of time.

In audio presentations, participants listen to a sentence and a word flashes on the screen at a particular point. Individual, unrelated sentences have listened to one at a time. A pause or a symbol (such as a row of stars) is usually used to indicate a new sentence is about to begin.

In both auditory and visual task presentations, participants sit in front of a computer screen and are given instructions on how to perform a task. Wires are attached to their head for event-related potential readings. For eye-tracking tasks, participants' heads are immobilized so that their gaze can be read. During these types of tasks in lexical ambiguity resolution experiments, there is usually no interaction or feedback given by the computer. In addition, filler sentences are often used to hide the purpose of the experiment.

In short, due to the fact that hypotheses must be tested in a controlled experimental setting, participants experience the visual and auditory presentation in a markedly different way than they do in naturally occurring conversations or reading or listening experiences in their everyday world. One way to overcome this artificiality would be to more closely mimic real-life environments, such as by having participants read longer passages of text (i.e., Mimicking webpage reading) or listen to an ongoing news program. While simulation of an ongoing conversation is even more difficult, it may be possible to watch clips of people engaged in conversation and test at points when lexical ambiguity is introduced. However, all these changes will make it more difficult to create and control stimuli, will take longer for the subjects to complete and will cost more to set up and run. In addition, future advances in virtual reality may make it possible to introduce a degree of interactivity into the experimental setting as well, but any degree of interactivity may well bring in variables that are extraneous to the task, which would then influence the resulting data to the extent that it no longer meets the criteria to run statistical analyses. It is this constraint, more than any other, that limits to what degree psychoor Neurolinguistic experiments can adequately test language processing in real-world contexts.

Task, probe position and timing: Task selection is constrained by the modality of presentation. As discussed above, reading tasks may involve word-byword reading or complete sentence or paragraph reading (Paragraph reading is not usually used in lexical ambiguity resolution tasks but is discussed below with relation to conceptual metaphor processing). Word-by- 


\section{J. Social Sci., 8 (3): 372-380, 2012}

word reading may vary in terms of where and how long the word is presented on the screen. In auditory tasks with a visual presentation (known as cross-modal presentation), the relevant factors involve the speed of the auditory presentation (fast or slow), the naturalness of the intonation, the type of task the participant is asked to do upon presentation of the visual probe, the position of the visual probe in relation to the ambiguity and the length of the visual probe presentation. The latter three issues are of particular concern in lexical ambiguity resolution experiments, although they do not usually discuss in any detail except to be reported in the methods section.

The first issue in cross-modal presentation concerns the task involved: when the word flashes on the screen while the participant is listening to the sentence, the subject either will need to decide if it is a word or not or say the word aloud. The reaction time between the presentation of the visual probe and the response (i.e., either a button press or the triggering of a voice key) is then measured. If the subject needs to decide if it is a word or not, s/he can press either the word or the non-word button (ideally with two fingers from the same hand so as to avoid handedness or hemispheric processing issues). In some lexical ambiguity experiments, however, a go/no-go method is used, where the participants only press a button if they see a word on the screen and they do nothing if they see a non-word (Tabossi and Zardon, 1993). However, this means that participants do not automatically perform an action upon seeing a stimulus; instead, they are deciding whether or not to perform an action, which inhibits the automaticity of the decision-making process (Hillert, 1998). In another type of cross-modal task known as a gating task, participants hear the sentence up to the ambiguous word and then hear a slight bit of the word, after which they guess at the word by writing it down and then listen to the sentence again, with a slightly longer piece of the ambiguous word presented auditorially and then they guess again. They follow this procedure until they guess the word (Li and Yip, 1998).

The issue raised by the go/no-go and gating tasks is whether or not the findings that lexical ambiguity resolution is context-dependent can be considered as valid. That is, the findings certainly show evidence for a context-dependent model. However, do the same findings necessarily argue against a contextindependent model? Given that a context-independent model must be tested under conditions where lexical access is automatic, do the go/no-go and gating tasks meet this criterion? I would argue that they both differ substantially from tasks (such as lexical decision tasks based on a yes or no button press, or naming) where there is no inhibition and where there is no guessing. In fact, all studies that point to a context-independent model involves cross-modal lexical decision (twobutton press) or cross-modal naming tasks.

Another assumption of the context-independent model has to do with the autonomy of lexical access. In order for the autonomy to be upheld, the visual probe presentation should, ideally, appear at the point of lexical access. If it appears later on, it is quite possible that exhaustive lexical access may have already occurred and that the processor is now at a new stage or in a new module where lexical selection is taking place. If the visual probe is presented after the point of lexical access, or if it is left on the screen for too long, or if it occurs at the end of a sentence where sentential wrapup effects occur, then the assumption of autonomy may have been violated and the context-independent model would not have been subject to a fair test.

However, these issues are often not discussed in any detail in the literature and each lab often uses its own methods and procedures without examining them to be sure that they are appropriate for the type of hypothesis testing that they are trying to perform.

In fact, a series of experiments (Hillert, 1998; Ahrens, 2001; 2006; Nakayama, 2002) has demonstrated that in cross-modal lexical decision tasks with the ambiguity occurring sentence medially and with the visual target presented no later than the offset of the ambiguity for $750 \mathrm{~m} \mathrm{Sec}$ or less, both primary and secondary meanings of nouns and verbs in Chinese are accessed even when the context is biased toward either the primary or secondary meaning of the ambiguous. These results support a context-independent model of lexical access and argue against a contextdependent model or a combined model incorporating contextual and lexical frequency factors.

In sum, tasks that require problem-solving on the part of participants, or tasks that involve visual presentation times of a lexical target that extend long after lexical access has been completed, provide evidence for context-dependent models of lexical ambiguity resolution. However, tasks that can be completed automatically and that have visual presentation times that do not extend long after lexical access has taken place provide evidence for contextindependent accounts. What is needed to advance our understanding of lexical ambiguity resolution further is the following:

a) A database indicating where lexical access takes place for words used in lexical ambiguity resolution experiments. This database ideally would include results from participants with high- 


\section{J. Social Sci., 8 (3): 372-380, 2012}

span and low-span working memory (and ideally could be sorted to look at either high-span working memory results, low-span working memory results, or the overall average of both groups.) This would allow researchers to first test the working memory span of their participants and modify their visual probe position accordingly

b) A database indicating the word senses for each lexical ambiguity and how strongly associated that sense is with the lexical item. This strength association could be calculated on the basis of the first sense a large sample of participants thinks of, or it could be based on the average percentage of that sense for all responses (Lin and Ahrens, 2010 for a further discussion of this issue and related literature). These participants might also be grouped into high and low verbal IQ, as low verbal IQ participants may generate fewer senses. Another way to calculate the strength of associated senses would be to analyze the data found in a large-scale corpus. The advantage of such a database would be the standardization of stimuli, such that a researcher could say that s/he picked the corpusbased analysis of the senses, or that s/he picked the first sense preference of low verbal IQ participants. This standardization would allow for easier comparisons across experiments

c) The selection of the modality, task, length of the visual target presentation, visual probe position all need to be not only specified (as they are now) but also given justification based on previous experimental findings and the expectations for current findings

d) Modality, task, length of the visual target presentation, visual probe position ideally should be varied across experiments (with the same stimuli and type of participants) in order to provide a degree of comparison that will allow for greater understanding of under what conditions contextdependent and context-independent processing takes place

While these four steps do not directly address the issues brought up on the section 'Modality' concerning the artificiality of the experimental setting in examining language processes, which are usually interactive in nature, they will make it easier for researchers to compare the robustness of their findings, which should lead to further advances in understanding issues related to the lexical ambiguity resolution.

Conceptual metaphor processing: Conceptual Metaphor Theory (Ortony, 1993; Lakoff and Johnson,
1980; 1999) postulates that metaphors are cognitive phenomena. They are instantiated in language, but they have conceptual underpinnings. Conceptual metaphors link two conceptual domains: a source domain and a target domain. A conceptual domain is a set of entities, qualities and functions that are semantically linked. The source domain usually consists of a concrete concept, such as MONEY, while the target domain involves an abstract concept, such as TIME. By convention, conceptual metaphors are written in small capitals in the form of X IS (A) Y, where X stands for the target domain and $\mathrm{Y}$ stands for the source domain.

The Conceptual Metaphor Theory postulates that we use the source domain to understand a target domain. For example, if we want to talk about TIME in Chinese (or in English) we can use the source domain of MONEY to do so (i.e., TIME IS MONEY), as in the example, "I don't want to spend any more time watching this stupid TV program." In this case, 'spending' is mapped from the source domain of MONEY to the target domain of TIME. We know about the meaning of 'spend' in the source domain that it means 'use up' and we apply this meaning to 'time' in the target domain.

The mappings between the source and target domain are systematic sets of correspondences that occur between concepts in the source and target domains. For example, in the conceptual metaphor LOVE IS A JOURNEY we find the following metaphorical usages in English, as in (6):

(6a): I don't know which direction our relationship is headed.

(6b): We'd better slow down and think about where we're going first.

(6c): I got lost in that relationship.

Researchers have long been interested in investigating whether or not the conceptual mappings underpinning conceptual metaphors are accessed and used in on-going discourse. Two major models have been proposed to account for the results found to date: the conceptual metaphor view and the attributive categorization view.

The conceptual metaphor view proposes that metaphors can be understood via mapping source/concrete domains to target/abstract domains, while the attributive categorization view suggests that there are no pre-existing mappings. Instead, metaphors are understood as a class-inclusion assertion, such that a metaphorical category is assigned to the source/concrete domain and this assignment entails a particular property of that category. As above, in the 


\section{J. Social Sci., 8 (3): 372-380, 2012}

case of lexical ambiguity resolution, both models have psycholinguistic data to back up their hypotheses. Prior studies on idioms (Gibbs, 1994), metaphors (Allbritton et al., 1995) and euphemisms (Pfaff et al., 1997) found evidence that conceptual mappings were accessed in a variety of experimental paradigms, including off-line rating, on-line priming and on-line reading experiments. In Nayak and Gibbs (1990) study, which asked participants to read and rate texts ending with one or two English idioms, prior context matched with one of the idioms but not the other. They found that the conceptually congruent condition was rated significantly more appropriate than the conceptually incongruent one, even though they were both contextually appropriate. However, Glucksberg et al. (1993) asked participants to perform a similar task in an online situation, where the prior context was presented in a line-by-line manner on the screen. Reading times for all the sentences could then be measured, up to and including the final sentence, which again contained one or two idioms. This on-line task did not find any evidence of facilitation for the conceptually congruent idiom as compared with the conceptually incongruent idiom. However, when Glucksberg et al. (1993) ran an off-line rating task similar to Nayak and Gibbs (1990) study, they did find that the conceptually congruent context facilitated the related idiom. Based on this data, they argued that while conceptual mappings may be accessed post-hoc, they are not used during on-line language processing.

Given our prior discussion concerning lexical ambiguity resolution, it is most likely apparent that there are differences between the experimental methods used: off-line rating and line-by-line reading. In fact, it is not a clear two-way distinction, as the two experiments differ in both presentation method and in the task. In the off-line rating task, the participants read a paragraph in its entirety and then make a decision after reading the last sentence. In the line-by-line reading task, the participants' only task is to press a button after they finish reading each sentence. At no point do they need to make a decision. In addition, it could be argued that the line-by-line presentation does not allow for a buildup of contextual clues, which would happen in a reading situation that more closely approximates a person's natural reading context (i.e., in paragraph format). Gong and Ahrens (2007) attempted to tease apart these issues by conducting five experiments. In Experiment 1, we tested whether conceptual mappings were accessed in an off-line paragraph judgment task (similar to the off-line rating task used by Nayak and Gibbs, 1990). Participants in this task were handed a document and asked to read paragraphs that had four or five instances of conceptual metaphors in each paragraph. Then they were asked to rate a target sentence that contained an instance from either the same conceptual mapping or a different conceptual mapping. Word frequency, collocating frequency and level of acceptability between the two terminal sentence conditions were controlled. In Experiment 2, an on-line paragraph judgment task was run that was similar to the off-line task, except that the participants read the paragraphs on a computer screen and made a yes-or-no judgment of the target metaphorical sentence. In Experiment 3, participants read the same materials as in Experiment 2, but they did not make any judgment on the final sentence. In Experiment 4, an on-line sentence judgment task, participants were asked to judge the final target sentence after viewing the preceding sentences line-byline on the computer screen. In Experiment 5, participants were asked to read the same materials with a line-by-line presentation on the screen.

Gong and Ahrens (2007) found that the mean rating scores for the terminal metaphor sentences in conceptually congruent pairings were significantly higher in both paragraph judging experiments (i.e., Experiments 1 and 2) but not in the line-by-line judging experiment (i.e., Experiment 4). Furthermore, the reading times were significantly faster for the conceptually congruent pairings than the non-congruent pairings in Experiment 2 (paragraph judging), but there was no difference in reading times in Experiment 4 (line-by-line judging). Furthermore, reading time for the target conceptually congruent sentence were faster in the paragraph experiment (Experiment 3) than in the non-paragraph experiment (Experiment 5).

These results suggest that conceptual mappings exist and are accessed in on-going discourse, such that when the target domain is built up throughout a paragraph and all the metaphors are taken from the same source domain, there is continuity in the conceptual processing, which allows for the facilitation as compared with the situation where the source domain changes and an adjustment then needs to be made. However, if a sentence is presented one line at a time on a screen, no build-up of global activation for semantic access of the concept mapping occurs, as the brain is processing each flash of visual information as a new item and, thus, no facilitation occurs for a visual target that involves the same source domain. This finding explains the discrepancy between the Nayak and Gibbs (1990) results and the Glucksberg et al. (1993) results, since Glucksberg et al. (1993) found no facilitation when they employed a line-by-line presentation. 
However, work still remains on determining if there is a minimum number of conventional metaphors necessary to activate conceptual mappings, or if the comparative frequency of the metaphors used will affect the degree of facilitation of the conceptual metaphor in the final target sentence. One issue of particular note in this regard concerns what Low et al. (2010) calls 'Mapping Principles' in the Conceptual Mapping Model. In this model, highly conventionalized conceptual metaphors are analyzed for frequent lexical examples of a conventional metaphor (Low et al., 2010). This analysis, which may be corpus-based (Gong et al., 2008; Ramesh and Kishore, 2007) or intuition-based, allows for a postulation of the reason underlying the source-target domain pairing (i.e., the conceptual metaphor). It further predicts that target domains will use different source domains for different reasons. Furthermore, analyzing mapping principles allows for additional predictions concerning reading times of target sentences. For example, one hypothesis is that if the target sentences are highly conventionalized, then whether or not they are preceded by metaphors that follow mapping principles will not influence reading times, but if the target sentences contain ambiguous or infrequent metaphors, then having the preceding context contain metaphors that follow mapping principles will facilitate reading times.

\section{CONCLUSION}

When hikers walk in unfamiliar territory, they take a compass and a map of the route they want to follow. They use the compass to orient themselves and then identify their location on a map. Similarly, when researchers ask a particular research question, it would be useful to orient themselves by asking why previous researchers found differing results and then identifying which potential methodological issues may be involved. Too often, researchers try to prove that their model handles additional hypotheses or accounts for different predictions. They spend a lot of time and money to prove that their model accounts for these different predictions and therefore that their model is superior. However, it is only when methodological issues in psycholinguistic processing (issues such as task selection, timing, stimuli creation, speed of audio presentation, size of visual characters/words) are resolved (or at least controlled) that the findings will be useful. Until then, any finding may simply be a case of researchers finding what they expect to see, simply because the task, methodology, or stimuli hold implicit biases that they are unaware of. If they step back, though and carefully survey the other landmarks on a parallel road, they may discover a theory that has greater explanatory value and forge pathways between the road they are on and the road alongside them, leading to greater explanatory adequacy and insight.

\section{ACKNOWLEDGEMENT}

I would like to extend my appreciation to students and colleagues who have worked with me and have discussed issues relating to lexical ambiguity resolution and conceptual metaphor processing, including Itesh Sachder, Tracy Love, Shu-ping Gong, Siaw-Fong Chung, Vicky Lai, Dora Lu and Chu-Ren Huang, along with many others. Special thanks go to David Swinney, who started me on the road of language processing and emphasized the critical importance of all aspects of methodology, including timing presentation and stimuli creation. All errors are my own responsibility.

\section{REFERENCES}

Ahrens, K., 2001. On-line sentence comprehension of ambiguous verbs in mandarin. J. East Asian Linguistics, 10: 337-358.

Ahrens, K., 2006. The effect of visual target presentation times on lexical ambiguity resolution. Language Linguistics, 7: 677-696.

Ahrens, K., L.L. Chang, K.J. Chen and C.R. Huang, 1998. Meaning representation and meaning instantiation for Chinese nominals. Comput. Linguistics Chinese Language Process., 3: 45-60.

Allbritton, D.W., G. McKoon and R.J. Gerrig, 1995. Metaphor-based schemas and text representations: Making connections through conceptual metaphors. J. Exp. Psychol.: Learn. Memory Cognition, 21: 612-625. DOI: 10.1037/02787393.21.3.612

Altarriba, J. and J.L. Gianico, 2003. Lexical ambiguity resolution across languages: A theoretical and empirical review. Exp. Psychol., 50: 159-170. DOI: 10.1026//1617-3169.50.3.159

Duffy, S.A., R.K. Morris and K. Rayner, 1988. Lexical ambiguity and fixation times in reading. J. Memory Language, 27: 429-446. DOI: 10.1016/0749-596X (88)90066-6

Fodor, J.A., 1983. The Modularity of Mind: An Essay on Faculty Psychology. 1st Edn., MIT Press, Cambridge, ISBN-10: 0262560259, pp: 145.

Gibbs, R.W., 1994. The Poetics of Mind: Figurative Thought, Language and Understanding. 1st Edn., Cambridge University Press, Cambridge, ISBN-10: 0521429927 , pp: 527. 
Giora, R., 1997. Understanding figurative and literal language: The graded salience hypothesis. Cognitive Linguistics, 8: 183-206.

Giora, R., 1999. On the priority of salient meanings: Studies of literal and figurative language. J. Pragmatics, 31: 919-929. DOI: 10.1016/S03782166(98)00100-3

Giora, R., 2003. On Our Mind: Salience, Context and Figurative Language. 1st Edn., Oxford University Press, New York, Oxford, ISBN-10: 0195136160, pp: 259.

Glucksberg, S., M. Brown and M.S. McGlone, 1993. Conceptual metaphors are not automatically accessed during idiom comprehension. Memory Cognition, 21: 711-719. DOI: 10.3758/BF03197201

Glucksberg, S., M.S. McGlone and D. Manfredi, 1997. Property attribution in metaphor comprehension. J. Memory Language, 36: 50-67.

Gong, S.P. and K. Ahrens, 2007. Processing conceptual metaphors in on-going discourse. Metaphor Symbol, 22: 313-330. DOI: 10.1080/10926480701528121

Gong, S.P. K. Ahrens and C.R. Huang, 2008. Chinese word sketch and mapping principles: A corpusbased study of conceptual metaphors using the BUILDING source domain. Int. J. Comput. Proces. Oriental Languages, 21: 3-17. DOI: 10.1142/S1793840608001755

Hillert, D., 1998. Sentence Processing: A Crosslinguistic Perspective. 1st Edn., Academic Press, San Diego, ISBN-10: 0126135312, pp: 437.

Hogaboam, T.W. and C.A. Perfetti, 1975. Lexical ambiguity and sentence comprehension. J. Verbal Learn. Verbal Behav., 14: 265-274. DOI: 10.1016/S0022-5371 (75)80070-3

Jones, L.L. and Z. Estes, 2005. Metaphor Comprehension as Attributive Categorization. J. Memory Language, 53: 110-124. DOI: 10.1016/j. jml. 2005.01.016

Keysar, B., Y. Shen, S. Glucksberg and W.S. Horton, 2000. Conventional Language: How Metaphorical is it? J. Memory Language, 43: 576-593. DOI: 10.1006/jmla.2000.2711

Lakoff, G. and M. Johnson, 1980. The metaphors we Live by. 2nd Edn., Chicago University Press, Chicago, ISBN-10: 0226468011, pp: 242.

Lakoff, G. and M. Johnson, 1999. Philosophy in the Flesh: The Embodied Mind and its Challenge to Western Thought. 1st Edn., Basic Books, New York, ISBN-10: 0465056741, pp: 624.

Li, P. and M.C. Yip, 1998. Context effects and the processing of spoken homophones. Read. Writ.: Interdisciplinary J., 10: 223-243. DOI: 10.1023/A:1008091816322
Lin, C.J.C. and K. Ahrens, 2010. Ambiguity Advantage Revisited: Two meanings are better than one when accessing Chinese nouns. J. Psycholinguistic Res., 39: 1-19. DOI: 10.1007/s10936-009-9120-8

Low, G., A. Deignan and Z. Todd, 2010. Researching and Applying Metaphor in the Real World. 1st Edn., John Benjamins Publishing Company, Amsterdam, ISBN-10: 9027223807, pp: 385.

Marslen-Wilson, W. and L.K. Tyler, 1980. The temporal structure of spoken language understanding. Cognition, 8: 1-71. DOI: 10.1016/0010-0277 (80)90015-3

Martin, C., H. Vu, G. Kellas and K. Metcalf, 1999. Strength of discourse context as a determinant of the subordinate bias effect. Q. J. Exp. Psychol. Section A: Hum. Exp. Psychol., 52: 813-839. DOI: 10.1080/713755861

McClelland, D.C., 1987. Human Motivation. 1st Edn., CUP Archive, New York, ISBN: 0521369517, pp: 663.

McClelland, J.L. and D.E. Rumelhart, 1981. An interactive activation model of context effects in letter perception: I. An account of basic findings. Psychol. Rev., 88: 375-407. DOI: 10.1037/0033295X.88.5.375

McGlone, M.S., 1996. Conceptual metaphors and figurative language interpretation: Food for thought? J. Memory Language, 35: 544-565.

Nakayama, M., 2002. Sentence Processing in East Asian Languages. 1st Edn., CSLI Publications, Stanford, ISBN-10: 1575863081, pp: 292.

Nayak, N.P. and R.W. Gibbs, 1990. Conceptual knowledge in the interpretation of idioms. J. Exp. Psychol. General, 19: 315-330. DOI: 10.1037/0096-3445.119.3.315

Noveck, I.A. and D. Sperber, 2006. Experimental Pragmatics. 1st Edn., Palgrave MacMillan, Basingstoke, ISBN-10: 1403903514, pp: 320.

Onifer, W. and D.A. Swinney, 1981. Accessing lexical ambiguities during sentence comprehension: Effects of frequency of meaning and contextual bias. Memory Cognition, 9: 225-236. DOI: 10.3758/BF03196957

Ortony, A., 1993. Metaphor and Thought. 2nd Edn., Cambridge University Press, Cambridge, ISBN-10: 0521405610, pp: 678.

Peleg, O., R. Giora and O. Fein, 2001. Salience and context effects: Two are better than one. Metaphor Symbol, 16: 173-192. DOI: 10.1080/10926488.2001.9678894

Pfaff, K.L., R.W. Gibbs Jr. and M.D. Johnson, 1997. Metaphor in using and understanding euphemism and dysphemism. Applied Psycholinguistics, 18: 59-83. 
Ramesh, R. and R. Kishore, 2007. Ontologies: A Handbook of Principles, Concepts and Applications in Information Systems. 1st Edn., Springer, New York, ISBN-10: 0387370196, pp: 930.

Swinney, D.A., 1979. Lexical access during sentence comprehension: ( $\mathrm{Re})$ consideration of context effects. J. Verbal Learn. Verbal Behav., 18: 645659. DOI: 10.1016/S0022-5371 (79)90355-4
Tabossi, P. and F. Zardon, 1993. Processing ambiguous words in context. J. Memory Language, 32: 359372. DOI: 10.1006/jmla. 1993.1019

Vu, H., G. Kellas and S.T. Paul, 1998. Sources of sentence constraint on lexical ambiguity resolution. Memory Cognition, 26: 979-1001. DOI: 10.3758/BF03201178 